\title{
GAIA Small for Gestational Age Level of Diagnostic Certainty Terminology
}

National Cancer Institute

\section{Source}

National Cancer Institute. GAIA Small for Gestational Age Level of Diagnostic Certainty

Terminology. NCI Thesaurus. Code C128703.

A subset of terminology related to small for gestational age, developed by the Global Alignment of Immunization safety Assessment in pregnancy consortium to aid in monitoring and improving fetal and maternal outcomes. 